\title{
Модификация поверхности GaAs и наблюдение эффекта гигантского рамановского рассеяния после диффузии индия
}

\author{
(C) А.С. Власов ${ }^{1}$, Л.Б. Карлина ${ }^{1}$, Ф.Э. Комисаренко ${ }^{2,3}$, А.В. Анкудинов ${ }^{1,2}$ \\ ${ }^{1}$ Физико-технический институт им. А.Ф. Иоффре Российской академии наук, \\ 194021 Санкт-Петербург, Россия \\ ${ }^{2}$ Университет ИТМО, \\ 197101 Санкт-Петербург, Россия \\ ${ }^{3}$ Санкт-Петербургский Академический университет, \\ 194021 Санкт-Петербург, Россия \\ E-mail: vlasov@scell.ioffe.ru
}

(Получена 17 ноября 2016 г. Принята к печати 21 ноября 2016 г.)

\begin{abstract}
Представлены результаты исследований поверхности GaAs в присутствии сурфактантов индия и фосфора. Показано, что в результате их диффузии (отжига) при температуре $650-670^{\circ} \mathrm{C}$ в приповерхностной области GaAs образуются кластеры, обогащенные индием. Кластеры проявляются в виде светлых пятен в изображении, полученном с помощью внутрилинзового детектора на сканирующем электронном микроскопе. В то же время исследования морфологии этой поверхности с помощью атомно-силового микроскопа обнаружили уменьшение значения среднеквадратичной шероховатости поверхности после процесса отжига (диффузии), что указывает на внедрение атомов индия в кристаллическую решетку GaAs. Кластеры ответственны за изменения в спектрах рамановского рассеяния: наблюдаются увеличение интенсивности сигнала, вызванное эффектом поверхностно-усиленного (гигантского) рамановского рассеяния, и сдвиг частоты колебаний в приповерхностной области. Обнаружено, что эффект формирования кластеров обусловлен как кристаллографической ориентацией поверхности, так и технологическими условиями ее подготовки.
\end{abstract}

DOI: 10.21883/FTP.2017.05.44461.8453

\section{1. Введение}

В последние годы влиянию условий термического отжига на состояние поверхности полупроводника уделяется довольно много внимания $[1,2]$. Этот интерес значительно усилился в последнее десятилетие в связи с бурным развитием приборов на основе наногетероструктур. Процессы первоначального формирования и роста квантовых слоев и точек, а также свойства приборов на их основе в значительной степени определяются рельефом поверхности, а также дефектами, формирующимися в процессе отжига $[3,4]$. Например, было показано, что подвижность электронов в InAs нанопроволоках зависит от шероховатости поверхности [5], или излучательная способность GaAs фотокатода зависит от состояния поверхности [3]. Известно, что шероховатость поверхности зависит от температуры отжига, среды, в которой этот отжиг осуществляется, а также от присутствия сурфактантов на поверхности во время отжига [4].

В роли сурфактантов, как правило, выступают примеси, изовалентные по отношению к материалу матрицы. Например, было показано, что присутствие индия в процессе роста слоев $\mathrm{AlGaAs}$ существенно улучшает состояние гетерограницы GaAs/AlAs. Это, в свою очередь, увеличивает подвижность электронов в $\mathrm{AlGaAs} / \mathrm{GaAs}$ I-HEMT [6].

Ранее нами была обнаружена существенная роль индия в процессах диффузии цинка в GaAs, а также влияние In и $\mathrm{P}$ на свойства гетероструктуры Ge-GaAs $[7,8]$.
В частности, было показано, что индий отвечает за глубину диффузии цинка, в то время как присутствие фосфора обеспечивает пассивацию поверхности.

Анализируя влияние сурфактантов и те процессы, которые происходят при отжиге арсенида галлия в различных средах, необходимо исследовать не только поверхность образца, но и те изменения, которые происходят в приповерхностной области материала. В данной работе представлен анализ поверхности арсенида галлия после отжига в присутствии индия и фосфора, а также характеристика приповерхностной области GaAs.

\section{2. Подготовка образцов}

Стандартная обработка поверхности GaAs перед процессом отжига включала химическую очистку в изопропиловом спирте с целью обезжиривания. Подложки GaAs отжигались в потоке водорода, насыщенного индием и фосфором. Температура отжига составляла 650-670 ${ }^{\circ}$. Время отжига менялось от 1 до 4 ч. Детали эксперимента подробно описаны в предыдущей статье [7]. Использовались подложки $n$-типа проводимости, легированные кремнием. Уровень легирования составлял $2-4 \cdot 10^{18} \mathrm{~cm}^{-3}$. Были исследованы epi-ready подложки, точно ориентированные (100) и разориентированные на угол 6 градусов в сторону направления [110], а также подложки, прошедшие обычную химико-механическую полировку ориентации (100). 


\section{3. Результаты и обсуждение}

Были исследованы рамановские спектры образцов до и после отжига. Спектры были получены при возбуждении одночастотным лазером с длиной волны 532 нм, работающим в непрерывном режиме. Облучение и сбор рассеянного света производилось через $50 \times$ объектив, расположенный перпендикулярно к изучаемой поверхности. Мощность излучения на образце была равна 4 мВт, для того чтобы избежать ошибок, вызванных возрастанием плотности фотовозбужденных носителей заряда. Спектры измерялись в геометрии обратного рассеяния в поляризации $z(x y) \bar{z}$. Согласно правилам отбора, в этой конфигурации в рамановских спектрах GaAs могут наблюдаться только продольные оптические (LO) фононы. На рис. 1 показаны рамановские спектры образца до (1) и после (2) диффузии индия. В спектре присутствуют три моды: LO $\sim 292 \mathrm{~cm}^{-1}$ соответствующая продольным оптическим колебаниям из приповерхностной области объемного заряда, $L_{-} \sim 268 \mathrm{~cm}^{-1}-$ смешанное фонон-плазмонное колебание и мода $L_{+}$, соответствующая колебаниям свободных электронов. Положения мод $L_{-}$и $L_{+}$определяются концентрацией свободных электронов. В отличие от LO-полосы, моды $L_{+}$и $L_{-}$ связаны с объемом GaAs. Глубина исследуемого слоя ограничивается глубиной поглощения и составляет порядка 100 нм для зеленого света (532 нм) в GaAs. Положение полосы $L_{+}$практически не зависит от обработки. Небольшой сдвиг может наблюдаться при исследовании образцов после длительного 4-часового отжига. Это означает, что в объеме материала не происходит существенных изменений: концентрация электрически активных дефектов $V_{\mathrm{As}}$ или $V_{\mathrm{Ga}}$ после отжига является незначительной. Основные изменения наблюдались в поведении LO-полосы: она сдвигалась в сторону меньших энергий, и ее относительная интенсивность (в сравнении с интенсивностью $L_{-}$-полосы) уменьшалась. Такое поведение спектров свидетельствует о модификации поверхностного слоя образца во время отжига. Относительное уменьшение интенсивности LO-полосы и ее сдвиг могут быть вызваны как внедрением инородных атомов в приповерхностную область объемного заряда (15-20 нм), так и создаваемыми из-за этого напряжениями.

На рис. 2 представлено изображение, полученное с помощью внутрилинзового детектора (обратнорассеянные электроны) на сканирующем электронном микроскопе. Исследования проводились на установке Zeiss Neon 40 EsB при ускоряющем напряжении 5 кВ. Глубина, с которой происходит получение сигнала при таком напряжении, составляет приблизительно 15 нм.

Изображение, полученное с помощью электронов, рассеянных назад, чувствительно к химическому составу: более тяжелые атомы более эффективно отражают падающие электроны. На изображении образца можно увидеть светлые пятна размером порядка $10 \mathrm{Hм}$ в диаметре, равномерно распределенные по плоскости

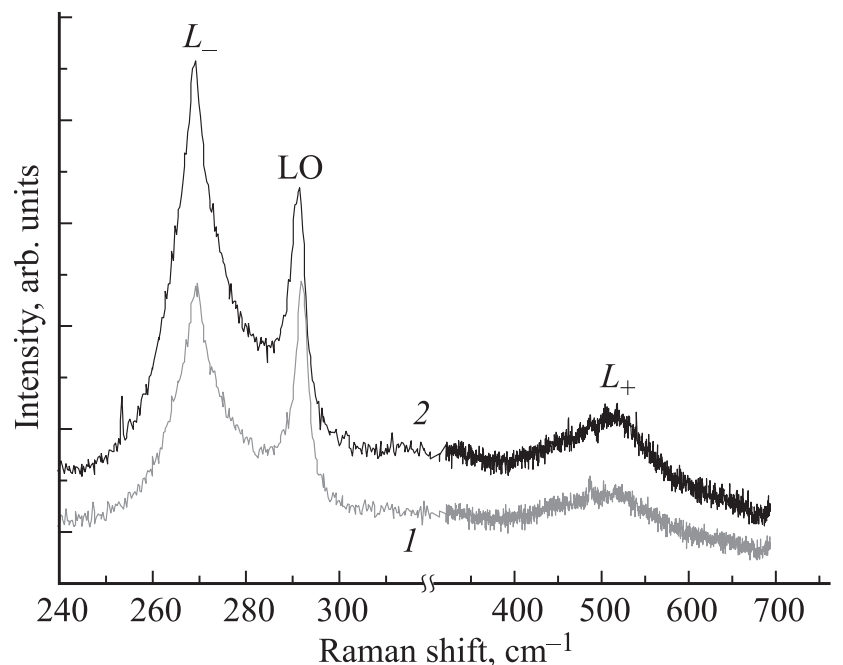

Рис. 1. Рамановские спектры образца GaAs до (1) и после (2) диффузии индия.

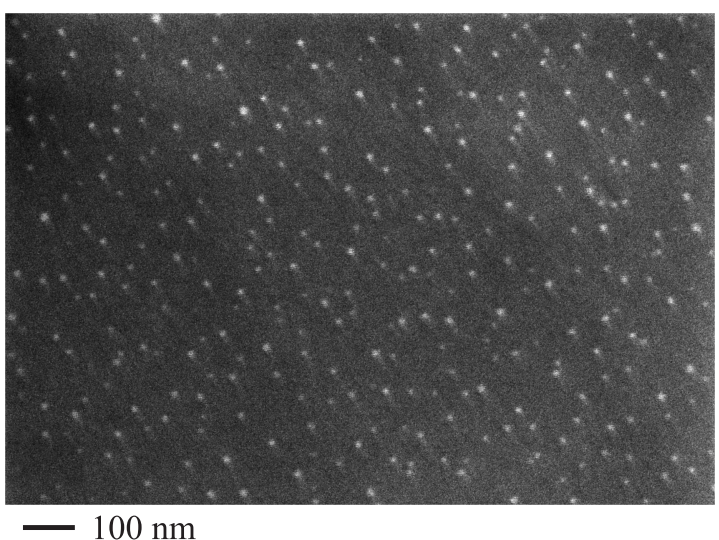

Рис. 2. СЭМ изображение (внутрилинзовый детектор) поверхности образца $\mathrm{GaAs}$ после диффузии In.

подложки. Таким образом, светлые пятна могут ассоциироваться с присутствием атомов индия.

Изображения тех же участков образца, полученные с помощью детектора вторичных электронов, не обнаружили формирования какого-либо значимого рельефа на поверхности образцов после отжига, т.е. контраст, наблюдаемый на рис. 2, образуется в результате внедрения атомов индия в решетку GaAs. Для проверки этого факта и для изучения состояния поверхности после отжига были проведены исследования с помощью атомносилового микроскопа. Шероховатость поверхности исследовалась с помощью установки NT-MDT Integra-Aura. Измерения проводились кантиливерами типа HA_NC в контактной моде и в режиме прерывистого контакта. Использование режима прерывистого контакта позволяет детектировать не только поверхностный рельеф кристалла. Давление кантиливера в этом режиме намного меньше, чем при использовании контактной моды, что позволяет определить присутствие на поверхности не 
только рельефных особенностей кристалла, но также наличие слабо связанных с поверхностью загрязнений. Для исследуемого региона, как правило, наблюдалось совпадение изображений в обоих режимах.

Для всех подложек наблюдался эффект снижения шероховатости поверхности после отжига: значения среднеквадратичного отклонения (параметр, характеризующий шероховатость поверхности) epi-ready подложек составили $0.2-0.3 \mathrm{Hм}$, тогда как для полированных образцов наблюдались величины $0.75-0.8$ нм. Таким образом, АСМ измерения подтверждают, что поверхность GaAs не деградирует в результате отжига, и на ней не происходит образования новых структур, а атомы индия внедряются в кристаллическую решетку с образованием кластеров.

В спектрах рамановского рассеяния можно обнаружить необычное усиление сигнала в образцах после диффузии. При этом отожженные образцы демонстрировали увеличение отражательной способности на несколько процентов. Таким образом, усиление рамановского сигнала не является оптическим артефактом, вызванным просветлением поверхности. Наблюдаемое поведение образцов после отжига можно отнести к так называемому эффекту гигантского рамановского рассеяния (SERS, Surface-Enhanced Raman Scattering). Обычно этот эффект наблюдается на подложках, содержащих металлические наночастицы, которые модифицируют локальное электрическое поле и усиливают взаимодействие поверхностных плазмонов со светом [9]. Усиление рамановского сигнала благодаря SERS-эффекту определяется расстоянием между частицами и может достигать нескольких порядков по интенсивности. Более короткие расстояния между частицами приводят к большим значениям локального электрического поля и, следовательно, к большему коэффициенту усиления рассеяния. В нашем случае в роли наночастиц выступают обогащенные In кластеры. Мы считаем, что модуляция поверхностного состава за счет пиннинга уровня Ферми приводит к образованию локальных заряженных состояний на поверхности кристалла и появлению периодического

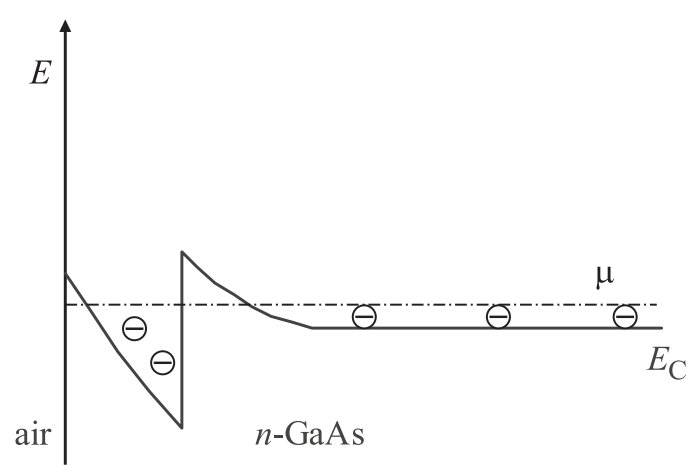

Рис. 3. Схематическое изображение зонной диаграммы GaAsподложки с кластерами, обогащенными In на поверхности (сплошная линия), и без них (пунктирная линия).
Относительное изменение интенсивности рамановского рассеяния в зависимости от типа подложки

\begin{tabular}{l|c|c|c}
\hline \multicolumn{1}{c|}{ Тип подложки } & epi-ready & epi-ready & $\begin{array}{c}\text { Механически } \\
\text { полированные }\end{array}$ \\
\hline $\begin{array}{l}\text { Отклонение } \\
\text { от направления [001] }\end{array}$ & $0^{\circ}$ & $6^{\circ}$ & $0^{\circ}$ \\
\hline $\begin{array}{l}\text { Относительная } \\
\text { интенсивность } \\
I \text { (difused)/I(reference) }\end{array}$ & 1.06 & 1.69 & 1.64 \\
\hline
\end{tabular}

потенциала. Схематически это проиллюстрировано на рис. 3. Ввиду того, что граница поверхностной области объемного заряда должна отражать общую потребность в компенсирующем заряде, она выравнивается, тогда как в поперечном направлении области, обогащенные In, получают отрицательный заряд. Другими словами, избыточное электрическое поле, которое образуется под кластерами, обогащенными In, притягивает к ним отрицательный заряд. Как видно из рис. 2, кластеры распределены со средним расстоянием 5-10 диаметров, делая эффект усиления незначительным. Также очевидно, что степень локализации поверхностных электронов должна быть незначительной ввиду малой концентрации индия и небольших размеров кластеров.

Косвенно, наличие поверхностного заряда в отожженных образцах подтверждается изменениями спектров отражения: в длинноволновой (подзонной) части спектра $(900-1100$ нм) отожженных образцов сохраняется отражательная способность на уровне $20-25 \%$. Описанные результаты могут иметь ту же природу, что и наблюдения сверхпроводимости в GaAs после диффузии индия [10].

Были исследованы образцы с различной ориентацией и обработкой поверхности. Эффекты модификации рамановских спектров наблюдались во всех отожженных образцах, однако на точно ориентированных epi-ready подложках этот эффект оказался наименее ярко выражен. В таблице собраны данные относительной интенсивности LO- и $L_{-}$-полос, наблюдаемых в рамановских спектрах (связанных с колебаниями решетки) после диффузии. Ввиду того что уровень легирования образцов с разными ориентациями немного отличался, интенсивность каждого спектра отнормирована по интенсивности к спектрам таких же необработанных образцов. Из таблицы видно, что увеличение интенсивности спектра точно ориентированной epi-ready подложки составляет 1.06, что находится в пределах погрешности измерений, тогда как на разориентированных и механически полированных подложках (т.е. подложках с большим количеством нуклеационных центров) наблюдается заметное увеличение интенсивности в $1.6-1.7$ раза.

Мы полагаем, что наблюдаемая модификация поверхности, приводящая к образованию кластеров, обогащенных In, может являться причиной для изменения 
механизма и скорости диффузии $\mathrm{Zn}$, наблюдаемой нами ранее [7]. Локальные напряжения от кластеров могут влиять на типы дефектов, образуемых в процессе диффузии, приводя к таким изменениям.

\section{4. Заключение}

Присутствие сурфактантов (In,P) и их дальнейшая диффузия приводят к существенной модификации поверхности и приповерхностной области GaAs образцов. Методом АСМ измерена среднеквадратичная шероховатость поверхности, значение которой уменьшалось после отжига.

В результате диффузии в приповерхностном слое образуются обогащенные индием кластеры диаметром $\sim 10$ нм. Модификация поверхности приводит к изменениям рамановских спектров, в которых наблюдается усиление сигнала, вызванное спонтанным SERS-эффектом. Данный эффект связан с начальным состоянием поверхности и уменьшается для образцов с меньшим количеством нуклеационных центров.

Работа выполнена при поддержке Российского научного фонда (грант № 14-29-00178).

\section{Список литературы}

[1] H. Morotaand, S. Adachia. J. Appl. Phys., 105, 123520 (2009).

[2] J.J.D. Lee, K.W. West, K.W. Baldwin, L.N. Pfeiffer. J. Cryst. Growth, 356, 46 (2012).

[3] S. Karkare, I. Bazarov, L. Cultrera, A. Iyer, X. Liu, W. Schaff. Proc. 2011 Particle Accelerator Conf. (Nrw York, NY, USA) THP192 (2011).

[4] D. Won, X. Weng, J.M. Redwing. Appl. Phys. Lett., 100, 021913 (2012).

[5] F. Wang, S.P. Yip, N. Han, K.W. Fok, H. Lin, J.J. Hou, G. Dong, T.F. Hung, K.S. Chan, J.C. Ho. Nanotechnology, 24, 375202 (2013).

[6] X.Z. Shang, W.C. Wang, S.D. Wu, Z.G. Xing, L.W. Guo, W.X. Wang, Q. Huang, J.M. Zhou. Semicond. Sci. Technol., 19, 519 (2004).

[7] L.B. Karlina, A.S. Vlasov, B.Y. Ber, D.Y. Kazantsev. J. Cryst. Growth, 432, 133 (2015).

[8] L.B. Karlina, A.S. Vlasov, B.Y. Ber, D.Y. Kazantsev, E.P. Marukhina. J. Cryst. Growth, 380, 138 (2013).

[9] K. Kneipp, M. Moskovits, H. Kneipp (Eds) SurfaceEnhanced Raman Scattering: Physics and Applications (Springer, 2006).

[10] J.M. Baranowski, P. Wojcik. Acta Phys. Polon. A, 82, 670 (1992).

Редактор А.Н. Смирнов

\section{GaAs surface modification and surface enhanced raman scattering observation after Indium diffusion}

\author{
A.S. Vlasov' ${ }^{1}$, L.B. Karlina ${ }^{1}$, Ph.E. Komissarenko, ${ }^{2,3}$, \\ A.V. Ankudinov ${ }^{1,2}$ \\ ${ }^{1}$ loffe Institute, \\ 194021 St. Petersburg, Russia \\ 2 ITMO University, \\ 197101 St. Petersburg, Russia \\ ${ }^{3}$ St. Petersburg Academic University, \\ 94021 St. Petersburg, Russia
}

\begin{abstract}
Characterization of the GaAs surface after annealing in a surfactant (Indium and Phosphorous) - rich atmosphere is presented. Indium-rich clusters are shown to be formed in the subsurface region after the annealing at $650-670^{\circ} \mathrm{C}$. They appear as bright spots in the scanning electron microscope image registered with an in-lens detector, while investigations of surface morphology by the atomic force microscopy characterization reveals a slight flattening of the surface indicating that In atoms are embedded in the lattice. These clusters are responsible for the changes observed in the Raman spectra, which reveal shifts due to the surface-layer-composition modification and demonstrate Raman signal amplification caused by a surface-enhanced Raman scattering effect. The effect of cluster formation is found to be connected with the initial surface condition: substrates with a higher number of nucleation centers (i. e., misoriented and nonepi-ready substrates) exhibit a higher degree of changes.
\end{abstract}

